\title{
Thermal Contact during the Cooling by the Single Roller Chill Block Casting
}

\author{
By Kunimasa Takeshita* and Paul Hideo Shingu**
}

\begin{abstract}
The thermal contact during the cooling of a Ni-19 at $\% \mathrm{P}$ alloy by the single roller chill block casting has been investigated by best fitting of experimentally measured actual temperatures of solidifying ribbons to calculations performed on the basis of heat transfer analysis. The effects of process variables on the thermal contact have been discussed in conjunction with contact patterns at the roller-side surface of the ribbons cast.
\end{abstract}

(Received October 21, 1985)

Keywords: casting, chill block melt spinning, rapid solidification, nickel-based alloy, nickelphosphorus alloy, amorphous, heat transfer coefficient

\section{Introduction}

Among various techniques of rapid quenching of molten metals and alloys, the single roller chill block casting is one of the commonest for its simplicity in facility and effectiveness in fabrication.

A large number of studies ${ }^{(1)-(5)}$ on the ribbon formation process by this technique have been developed from the view point of thermal and momentum transport within the puddle, the melt pool formed on the rotating substrate. In these analyses, it becomes important to know the value of heat transfer coefficient at ribbonroller interface for determining which transport mechanism is dominant.

Ruhl $^{(6)}$ has made a thermal analysis during the splat cooling and pointed out that the splat thickness and the quality of the thermal contact between the splat and substrate are the most important process variables for the cooling rates. Therefore, in order to make an analysis on the cooling or solidification process during the single roller chill block casting and to discuss the variation of the microstructure formed by this technique, it also becomes important to know the value of heat transfer

* Department of Mechanical Engineering, Faculty of Engineering, Fukui University, Fukui 910, Japan.

** Department of Metal Science and Technology, Faculty of Engineering, Kyoto University, Kyoto 606, Japan. coefficient.

Up to the present, some workers ${ }^{(7)-(9)}$ have reported the values of heat transfer coefficient during the single roller chill block casting. These values, however, vary considerably from experiment to experiment.

The purpose of the present work is to estimate the value of heat transfer coefficient by measuring actual temperatures of solidifying $\mathrm{Ni}-19$ at $\% \mathrm{P}$ alloy ribbons with an infrared thermometer and by best fitting of these experimentally measured temperatures to those of calculations performed on the basis of heat transfer analysis. The effects of three different roller materials and roller surface velocity on the value of heat transfer coefficient will also be discussed in conjunction with contact patterns at the roller-side surface of the ribbons cast.

\section{Experimental Procedures}

A Ni-P master alloy with the eutectic composition (Ni-19 at \% P), known as one of nickel based amorphous forming alloys, was prepared by induction melting of $99.9 \%$ pure nickel and $99.9 \%$ pure $\mathrm{Ni}-23.6$ at $\% \mathrm{P}$ alloy in an alumina crucible under a protective argon atmosphere and was chill cast in a carboncoated copper mold.

The alloy sample about $5 \mathrm{~g}$ in mass was melted in a quartz crucible $(10 \mathrm{~mm}$ in inner diameter and $1 \mathrm{~mm}$ in orifice diameter) at 1323 
$\mathrm{K}$ under argon atmosphere. The molten sample, being ejected by argon gas pressure on the circumferential surface of a $300 \mathrm{~mm}$ diameter roller, was rapidly solidified to a ribbon in open air. The nozzle bottom to roller surface spacing was set as $0.3 \mathrm{~mm}$. The roller materials chosen were copper, steel $(0.45$ mass $\%$ carbon) and type 304 stainless steel. The process variables of the argon gas ejection pressure, $P_{e}$, and the roller surface velocity, $U_{w}$, were varied within a good shaped and oxidation free ribbon sample forming range. These process variables were $P_{e}=49 \mathrm{kPa}$ and $U_{w}=17,23,33$, $43 \mathrm{~m} \cdot \mathrm{s}^{-1}$ for copper roller, $P_{e}=29 \mathrm{kPa}$ and $U_{w}=22,27,31 \mathrm{~m} \cdot \mathrm{s}^{-1}$ for steel roller and $P_{e}=20 \mathrm{kPa}$ and $U_{w}=31,36,43 \mathrm{~m} \cdot \mathrm{s}^{-1}$ for stainless steel roller. Prior to each run, the roller surfaces were prepared by abrasion with 800 grade carborundum paper.

Actual temperatures of a solidifying ribbon were directly measured by an infrared thermometer with an optical system $50 \mathrm{~mm}$ in focal distance and focus $1 \mathrm{~mm}$ in diameter. The temperature measurements were made one at a time by varying the setting distance down stream from the ejected point. The output from the infrared thermometer was recorded by using an oscilloscope with digital memory. The infrared thermometer was calibrated by watching a planar jet of the same alloy melt at $1273 \mathrm{~K}$ which was measured by an alumelchromel thermocouple.

For the calculation of cooling process, a photograph of a stationary melt puddle formed between nozzle bottom surface and roller surface was taken, and the average thickness of a ribbon cast was measured. The close-up picture of the melt puddle was made using a $100 \mathrm{~mm} \mathrm{f} 1: 4$ macro lens and a $100 \mathrm{~mm}$ extension tube. The exposure time was $8 \mathrm{~ms}$ throughout which was enough to be sufficiently exposed by the light of the melt puddle. The average ribbon thickness was determined by calculation from the ribbon mass divided by length, width and density.

In order to examine the contact between ribbon and roller surface, the roller-side surface of a ribbon cast was observed through a scanning electron microscope. The microstructural examination of a ribbon cast was also made through the scanning electron microscope after mechanical abrading and deep etching in 10\% aqueous $\mathrm{HNO}_{3}$.

\section{Results}

\section{Microstructure of a ribbon cast}

$\mathrm{Ni}-19$ at $\% \mathrm{P}$ alloys cast on steel and stainless steel rollers and on copper roller with a roller surface velocity of $17 \mathrm{~m} \cdot \mathrm{s}^{-1}$ showed some crystals growing from the ribbon bottom surface and quenched in the half way to the top surface, while the alloys cast on copper roller with roller surface velocities higher than 17 $\mathrm{m} \cdot \mathrm{s}^{-1}$ did not show any quenched-in crystals, as shown in Fig. 1.

\section{Actual temperatures of a solidifying ribbon}

The recorded output from the infrared thermometer showed first sharp increase followed by a relatively steady state and the final sharp drop. Then, the temperature in the relatively steady state was taken as the temperature of a solidifying ribbon at a setting distance down stream from the ejected point. In Figs. 2, 3 and 4 , the observed temperatures of solidifying ribbons cast on three different roller materials are plotted against the distance down stream from the end point of the melt puddle, which is defined as a setting distance plus $L_{3}$ as indicated in Fig. 5. The temperatures plotted in Figs. 2, 3 and 4 are determined by averaging several measurements at each setting distance.

\section{Estimation of the value of heat transfer coefficient}

From the measured actual temperatures of solidifying ribbons, the value of heat transfer coefficient at the metal-roller interface will be estimated by making an analysis of the cooling process during the single roller chill block casting. The analysis ${ }^{(5)}$ of the cooling process should involve a treatment of both thermal and momentum transport, especially within the melt puddle region. Since such treatment is too complicated to evaluate the heat transfer coefficient, some assumptions and simplifications are introduced. They are as follows: 

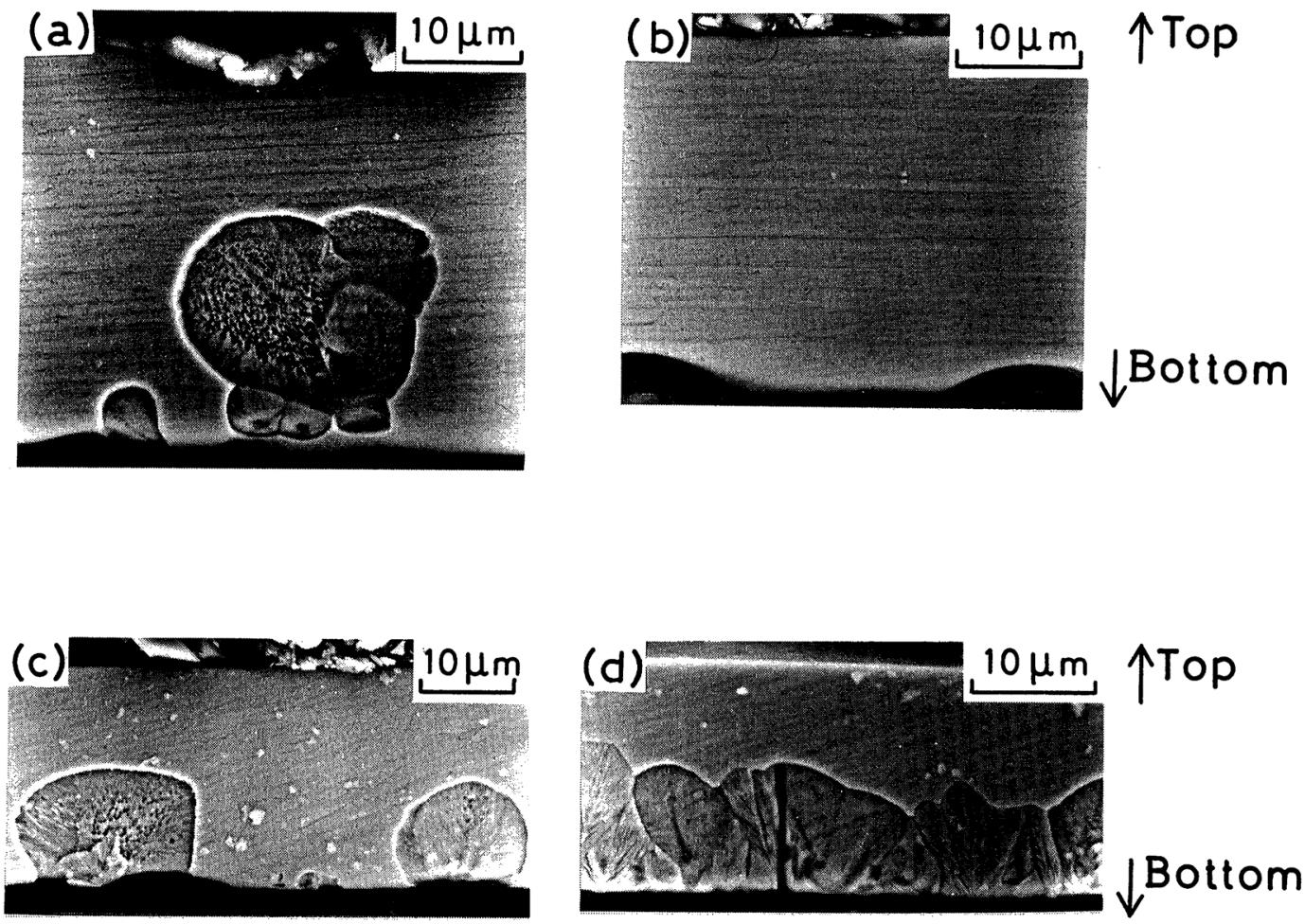

Fig. 1 SEM micrographs of cross sections of Ni-P alloys cast on (a) coper roller with $U_{w}=17$ $\mathrm{m} \cdot \mathrm{s}^{-1}$, (b) copper roller with $U_{w}=23 \mathrm{~m} \cdot \mathrm{s}^{-1}$, (c) steel roller with $U_{w}=27 \mathrm{~m} \cdot \mathrm{s}^{-1}$ and (d) stainless steel roller with $U_{w}=31 \mathrm{~m} \cdot \mathrm{s}^{-1}$.

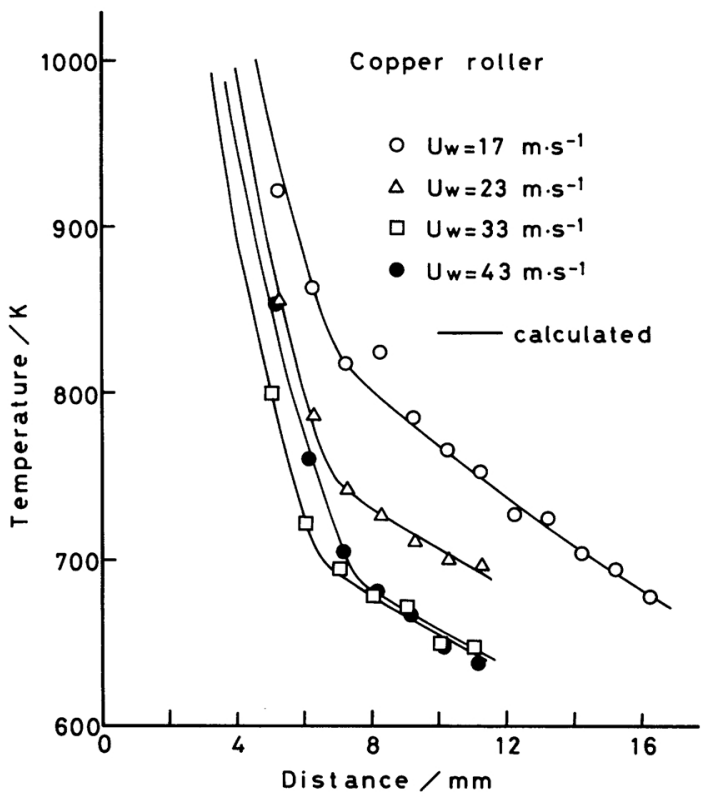

Fig. 2 Measured and calculated temperatures of Ni-P alloy ribbons cast on copper roller.

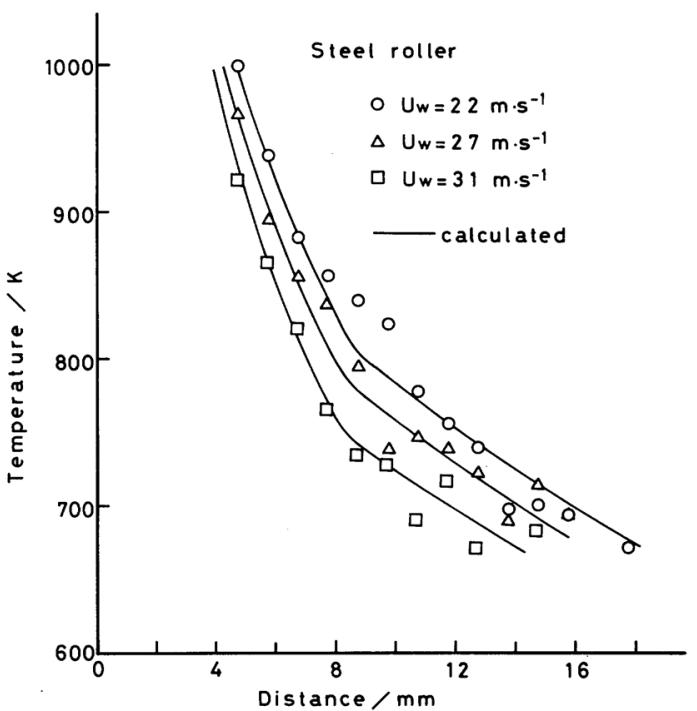

Fig. 3 Measured and calculated temperatures of $\mathrm{Ni}-\mathrm{P}$ alloy ribbons cast on steel roller. 


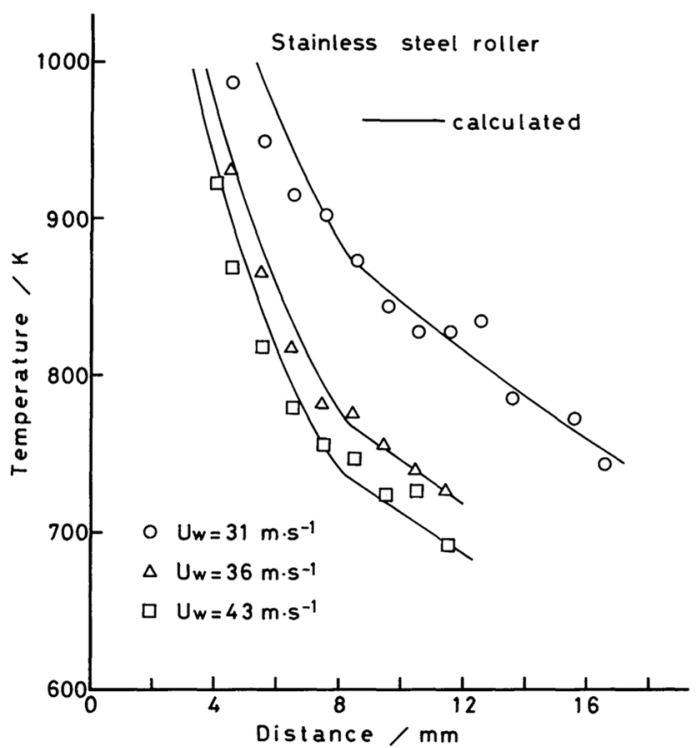

Fig. 4 Measured and calculated temperatures of Ni-P alloy ribbons cast on stainless steel roller.

(1) Heat transport by convective melt flow is negligible.

(2) Heat conduction pararell to the substrate surface is negligible.

(3) The loss of heat due to the radiation from free surface is small.

(4) There is no slip between the melt and the roller surface.

(5) Initial temperature distributions of roller substrate and melt puddle are uniform and kept at $T_{0}$ and $T_{m}$, respectively.

(6) Temperature at top surface of puddle is maintained at $T_{m}$.

Assumptions (1), (2) and (3) allow this problem to be treated as an one-dimensional heat flow problem. Referring to Fig. 5 and considering a co-ordinate system moving with the roller surface, the governing equation within the metal and the substrate regions will be described as

$$
\frac{\partial T}{\partial t}=\alpha \frac{\partial^{2} T}{\partial y^{2}},
$$

where $T=$ temperature,

$$
t=\text { time, }
$$$$
\alpha=\text { thermal diffusivity }
$$

and

$y=$ position variable, as indicated in Fig. 5 .

Initial conditions are

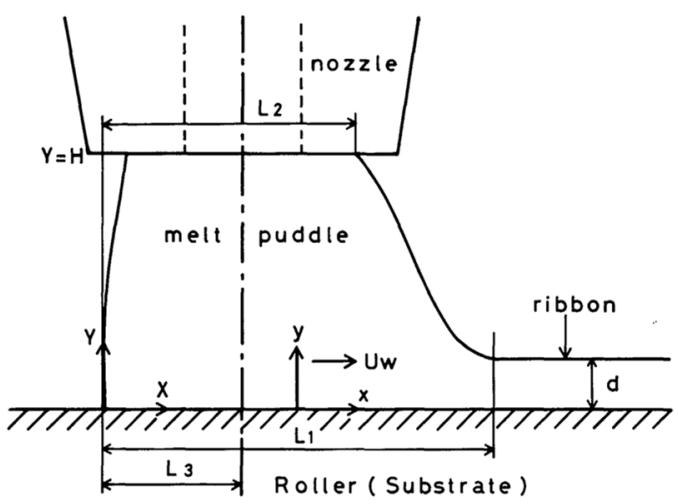

Fig. 5 Schematic representation of the problem and puddle length definition.

$$
T_{B}(y, 0)=T_{0} \text { for } y<0
$$

and

$$
T_{L}(y, 0)=T_{m} \text { for } 0 \leq y \leq H .
$$

Boundary conditions are,

(1) while the co-ordinate travels up to a distance $L_{2}$,

$$
T_{L}=T_{m} \quad \text { at } y=H,
$$

(2) while the co-ordinate travels from $L_{2}$ to $L_{1}$,

$$
\frac{\partial T_{L}}{\partial y}=0 \quad \text { at free surface, }
$$

(3) while the co-ordinate travels beyond $L_{1}$,

$$
\begin{aligned}
& \frac{\partial T_{L}}{\partial y}=0 \quad \text { at } y=d, \\
&\left.K_{L} \frac{\partial T_{L}}{\partial y}\right)_{y=0}=h\left(T_{L}(0, t)-T_{B}(0, t)\right) \\
& \text { at } y=0
\end{aligned}
$$

and

$$
T_{B}=T_{0} \text { at } y=-\infty .
$$

In the above equations

$T_{0}=$ initial substrate temperature,

$T_{m}=$ initial melt temperature,

$H=$ nozzle bottom surface-roller surface spacing,

$d=$ ribbon thickness,

$K_{L}=$ thermal conductivity of the metal, $h=$ heat transfer coefficient at the 
and

metal-roller interface

$L_{1}$ and $L_{2}=$ lengthes defining puddle shape as indicated in Fig. 5.

Subscripts $L$ and $B$ refer to the metal and substrate, respectively. Prior to calculation, it is necessary to obtain experimentally the puddle lengthes $L_{1}, L_{2}$ and $L_{3}$ and ribbon thickness $d$, which are summarized in Table 1 . Calculations are performed using an implicit finite difference numerical method. In the numerical method, the boundary condition (5) is treated in such a way that while the moving co-ordinate system travels from $L_{2}$ to $L_{1}$, a segment of the space cells in adjacent to the free surface is removed step by step resulting in the final space cells according to the ribbon thickness. The physical properties of the Ni-19 at $\% \mathrm{P}$ alloy and the three roller materials used for calculations are listed in Table 2 . The initial substrate temperature, $T_{0}$, and the initial melt temperature, $T_{m}$, are set to $293 \mathrm{~K}$ and $1323 \mathrm{~K}$, respectively.

Calculations enable to illustrate the temperature at the top surface of a solidifying ribbon as a function of the distance down stream from the end point of the melt puddle with a value of heat transfer coefficient, $h$, as a parameter. Hence, one can estimate the value

Table 1 Observed puddle lengthes and average ribbon thickness.

\begin{tabular}{lcccccccccc}
\hline \hline $\begin{array}{l}\text { Roller } \\
\text { material }\end{array}$ & \multicolumn{3}{c}{ Copper } & \multicolumn{4}{c}{ Steel } & \multicolumn{4}{c}{$\begin{array}{c}\text { Stainless } \\
\text { steel }\end{array}$} \\
\hline$U_{w}\left(\mathrm{~m} \cdot \mathrm{s}^{-1}\right)$ & 17 & 23 & 33 & 43 & 22 & 27 & 31 & 31 & 36 & 43 \\
\hline$L_{1}(\mathrm{~mm})$ & 3.6 & 3.4 & 2.7 & 2.7 & 2.9 & 2.4 & 2.4 & 2.3 & 2.2 & 2.1 \\
$L_{2}(\mathrm{~mm})$ & 2.8 & 2.9 & 2.3 & 2.3 & 2.2 & 1.8 & 1.7 & 1.7 & 1.6 & 1.5 \\
$L_{3}(\mathrm{~mm})$ & 1.3 & 1.3 & 1.0 & 1.1 & 0.79 & 0.79 & 0.69 & 0.61 & 0.46 & 0.51 \\
$d(\mu \mathrm{m})$ & 40 & 31 & 24 & 21 & 29 & 24 & 21 & 19 & 16 & 13 \\
\hline \hline
\end{tabular}

of $h$ by best fitting of the calculated curve of temperature change at the ribbon top surface to experimentally measured ribbon surface temperatures at various distances. Any calculated curve with a constant value of $h$ throughout the cooling can not, however, fit all measured ribbon surface temperatures. Therefore, in the present study the value of $h$ is changed in the midst of the cooling, in order that the best fitting may be made. The calculated best fitted curves are shown in Figs. 2, 3 and 4.

The distances at which the $h$ values are changed are $6 \mathrm{~mm}$ for the copper roller and 8 $\mathrm{mm}$ for the steel and stainless steel rollers, independent of roller surface velocities. The temperatures of ribbon bottom surfaces when the $h$ values are changed are plotted as a function of roller surface velocity, as shown in Fig. 6 . The $h$ value change in the midst of the cooling is due to the change in thermal contact between ribbon and roller surface. This sudden decrease in $h$ should not take place, when the ribbon bottom temperature is above $T_{g}$ in order to obtain fully amorphous ribbons. Ribbons cast on the steel and stainless steel rollers and on the copper roller with roller surface velocity of $17 \mathrm{~m} \cdot \mathrm{s}^{-1}$ showed calculated ribbon bottom surface temperatures at $h$ value change higher than the glass transition temperature, resulting in the appearance of some crystals observed by microstructual examinations.

The estimated values of heat transfer coefficient, denoted $h_{1}$ before $h$ value change and $h_{2}$ after $h$ value change, are summarized in Fig. 7. Both $h_{1}$ and $h_{2}$ values generally increase with the increse in roller surface velocity.

Table 2 Physical properties of Ni-19 at $\% \mathrm{P}$ alloy and roller materials used for calculations. The values of pure nickel in the liquid state are used for the properties of $\mathrm{Ni}-19$ at\% $\mathrm{P}$ alloy.

\begin{tabular}{|c|c|c|c|c|}
\hline Materials & & $\begin{array}{l}\text { Thermal conductivity } \\
\left(\mathrm{W} \cdot \mathrm{m}^{-1} \cdot \mathrm{K}^{-1}\right)\end{array}$ & $\begin{array}{l}\text { Specific heat } \\
\left(\mathrm{J} \cdot \mathrm{kg}^{-1} \cdot \mathrm{K}^{-1}\right)\end{array}$ & $\begin{array}{c}\text { Density } \\
\left(\mathrm{kg} \cdot \mathrm{m}^{-3}\right)\end{array}$ \\
\hline $\begin{array}{l}\mathrm{Ni}-19 \text { at } \% \mathrm{P} \\
\text { (molten } \mathrm{Ni} \text { ) }\end{array}$ & (sample) & $3.0 \times 10$ & $6.2 \times 10^{2}$ & $8.0 \times 10^{3}$ \\
\hline Copper & (substrate) & $4.0 \times 10^{2}$ & $3.9 \times 10^{2}$ & $9.0 \times 10^{3}$ \\
\hline Steel & (substrate) & $4.2 \times 10$ & $5.5 \times 10^{2}$ & $7.8 \times 10^{3}$ \\
\hline Stainless steel & (substrate) & $1.6 \times 10$ & $5.0 \times 10^{2}$ & $8.0 \times 10^{3}$ \\
\hline
\end{tabular}




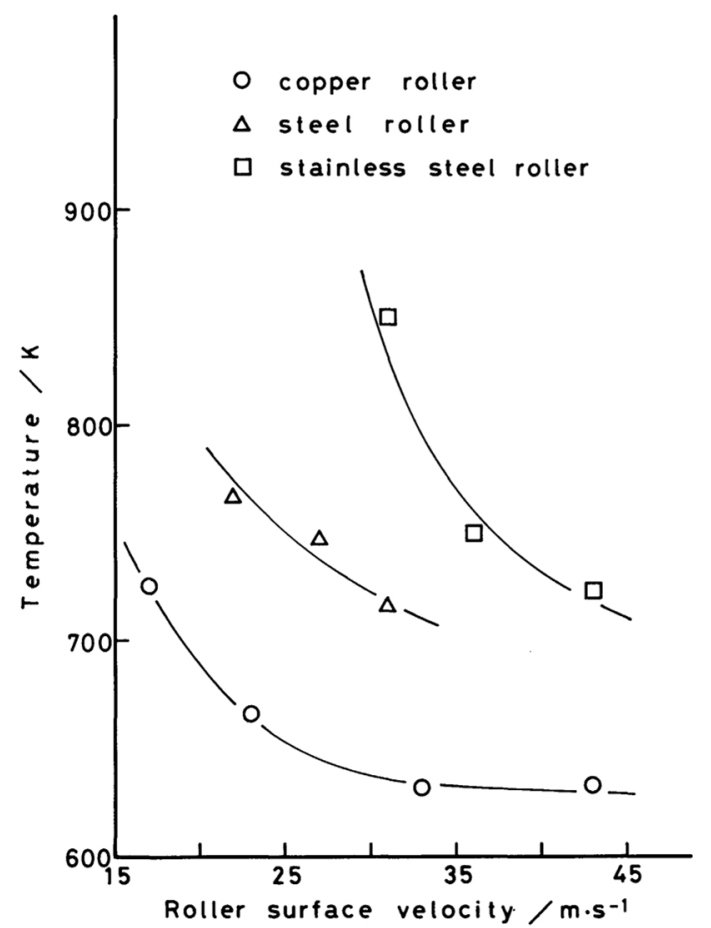

Fig. 6 Calculated temperatures of ribbon bottom surfaces at $h$ value change for three roller materials.

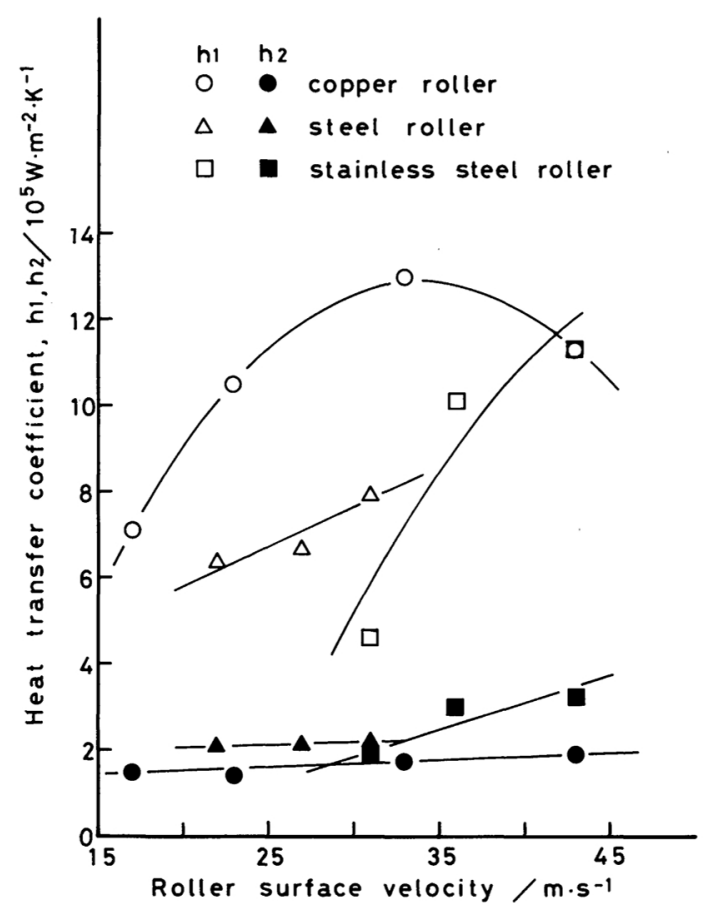

Fig. 7 Estimated values of heat transfer coefficients $h_{1}$ and $h_{2}$.

\section{Contact between ribbon and roller surface}

The contact pattern of a ribbon cast on the copper roller markedly varies with the roller surface velocity, as shown in Fig. 8. It can be seen from this figure that the size of air pockets incorporated during the casting increases with the decrese in roller surface velocity. The three roller materials used show some differences in the shape and size of air pockets, as shown in Fig. 9.

The mechanical contact between ribbon and roller surface can be estimated by calculating the contact area, defined as a percentage of the area subtracted by an air pocket occupied area to the total area, and the result is shown in Fig. 10. For the copper roller, the contact area linearly increases with the increase in roller surface velocity in contrast to the cases of other roller materials.

\section{Correlation between $h$ value and contact area}

It is expected that the mechanical contact between ribbon and roller surface affects the $h$ values at the ribbon-roller interface. Figure 11 shows the correlation between $h$ values and contact area. The $h_{1}$ values increase independent of roller materials in the range from $7 \times 10^{5} \mathrm{~W} \cdot \mathrm{m}^{-2} \cdot \mathrm{K}^{-1}$ to $1.3 \times 10^{6} \mathrm{~W} \cdot \mathrm{m}^{-2} \cdot \mathrm{K}^{-1}$ with the increase in contact area from $50 \%$ to $80 \%$, while $h_{2}$ values are almost constant at $2 \times 10^{5} \mathrm{~W} \cdot \mathrm{m}^{-2} \cdot \mathrm{K}^{-1}$ in spite of the increase in contact area. This result indicates that before $h$ value change the mechanical contact between the melt and the roller surface dominantly determines the thermal contact at the meltroller interface, while after $h$ value change the contribution of mechanical contact to the thermal contact becomes less important.

\section{Discussion}

The $h$ values during the single roller chill block casting, which have been estimated up to the present, are in the range from $5.0 \times 10^{5}$ $\mathrm{W} \cdot \mathrm{m}^{-2} \cdot \mathrm{K}^{-1}$ to $2.1 \times 10^{6} \mathrm{~W} \cdot \mathrm{m}^{-2} \cdot \mathrm{K}^{-1}$ after Huang and Fiedler ${ }^{(7)}, 8.4 \times 10^{5} \mathrm{~W} \cdot \mathrm{m}^{-2} \cdot \mathrm{K}^{-1}$ after Katgerman and Brink ${ }^{(8)}$ and $6 \pm 3 \times 10^{4}$ 

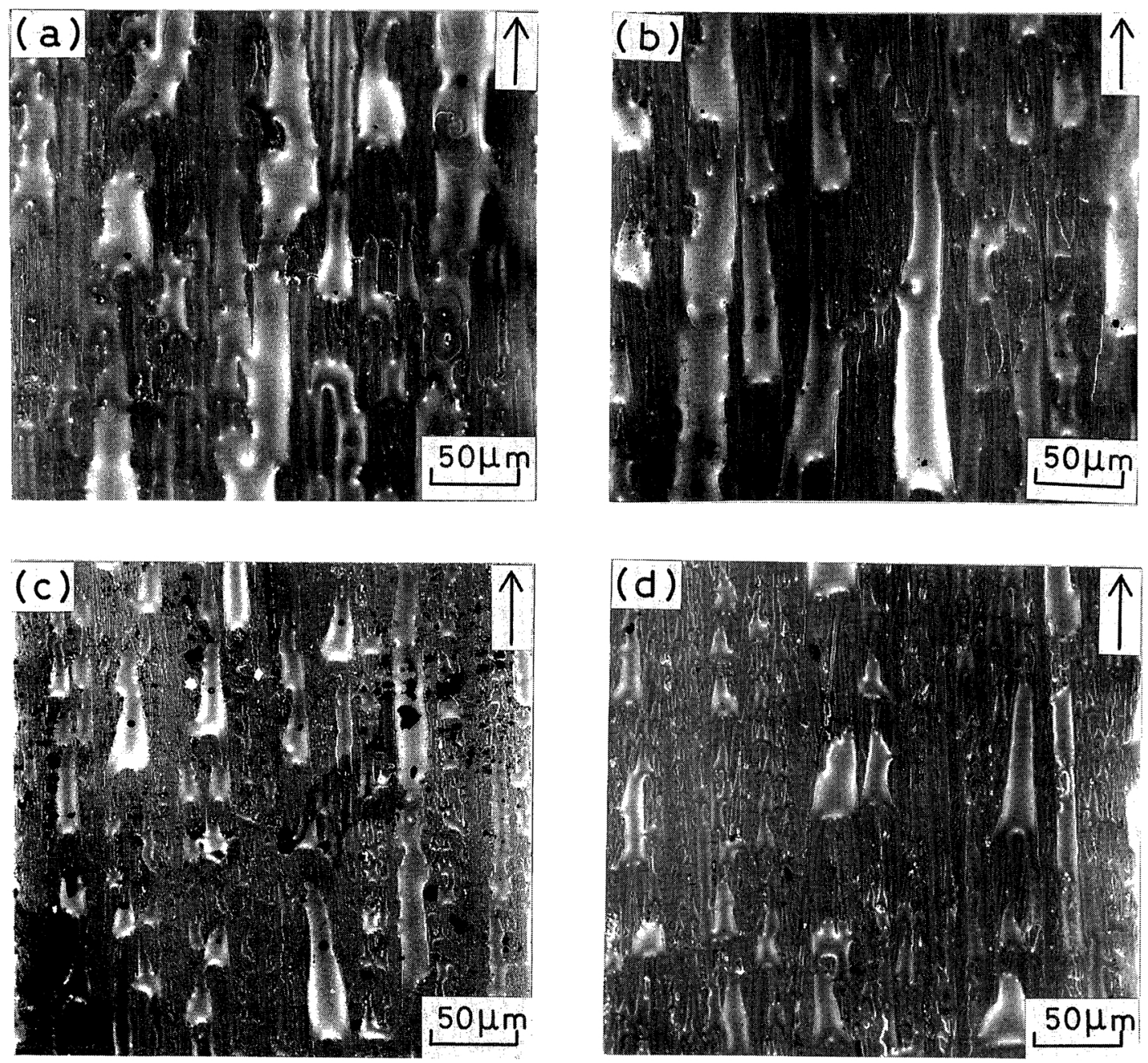

Fig. 8 SEM micrographs at the roller-side surfaces of Ni-P alloy ribbons cast on copper roller with (a) $U_{w}=17 \mathrm{~m} \cdot \mathrm{s}^{-1}$, (b) $U_{w}=23 \mathrm{~m} \cdot \mathrm{s}^{-1}$, (c) $U_{w}=33 \mathrm{~m} \cdot \mathrm{s}^{-1}$ and (d) $U_{w}=43 \mathrm{~m} \cdot \mathrm{s}^{-1}$. Arrow indicates the rotating direction.
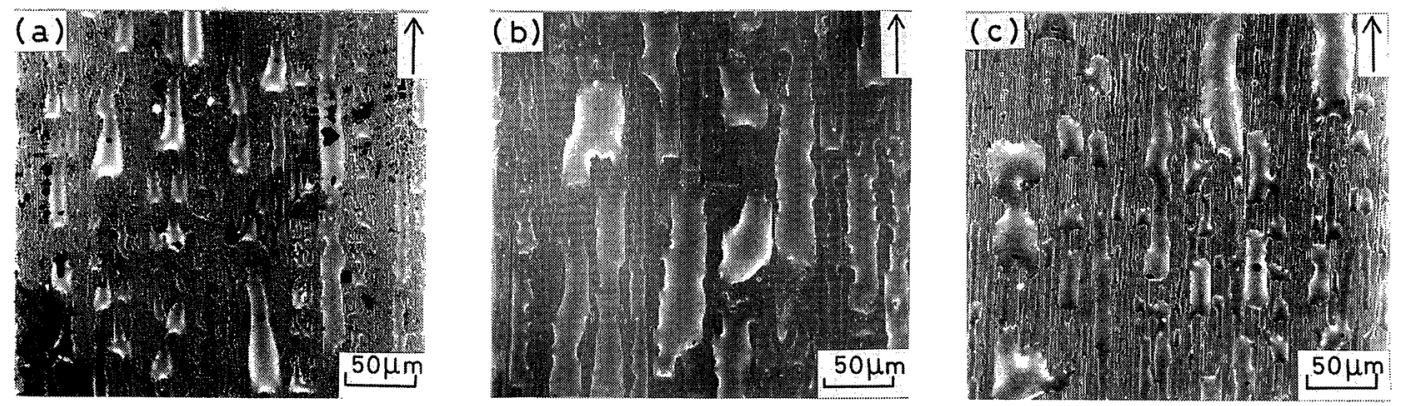

Fig. 9 SEM micrographs at the roller-side surfaces of Ni-P alloy ribbons cast on (a) copper roller with $U_{w}=33 \mathrm{~m} \cdot \mathrm{s}^{-1}$, (b) steel roller with $U_{w}=31 \mathrm{~m} \cdot \mathrm{s}^{-1}$ and (c) stainless steel roller with $U_{w}=31$ $\mathrm{m} \cdot \mathrm{s}^{-1}$. Arrow indicates the rotating direction. 


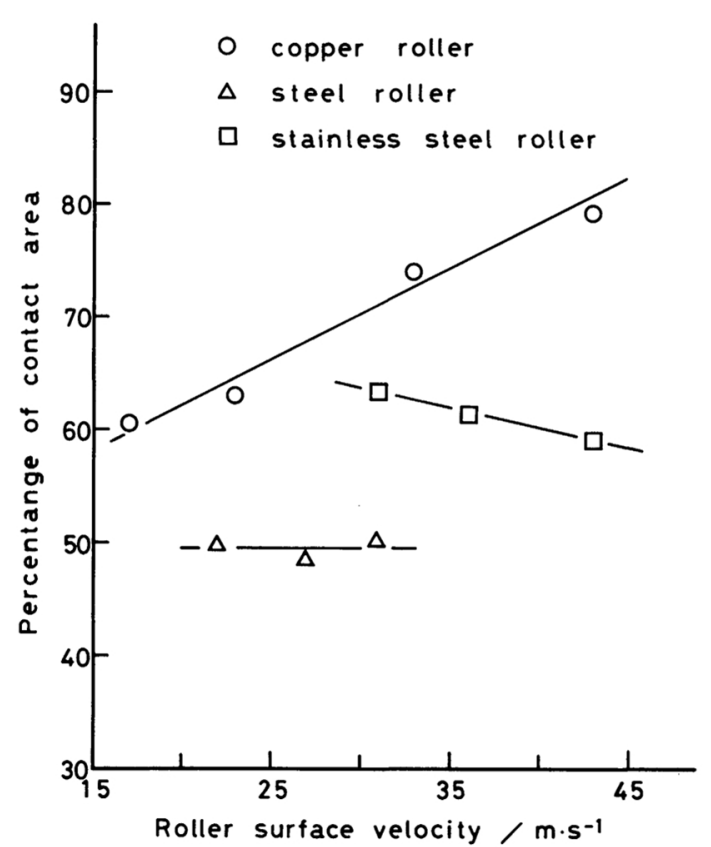

Fig. 10 Effect of roller surface velocity on contact area.

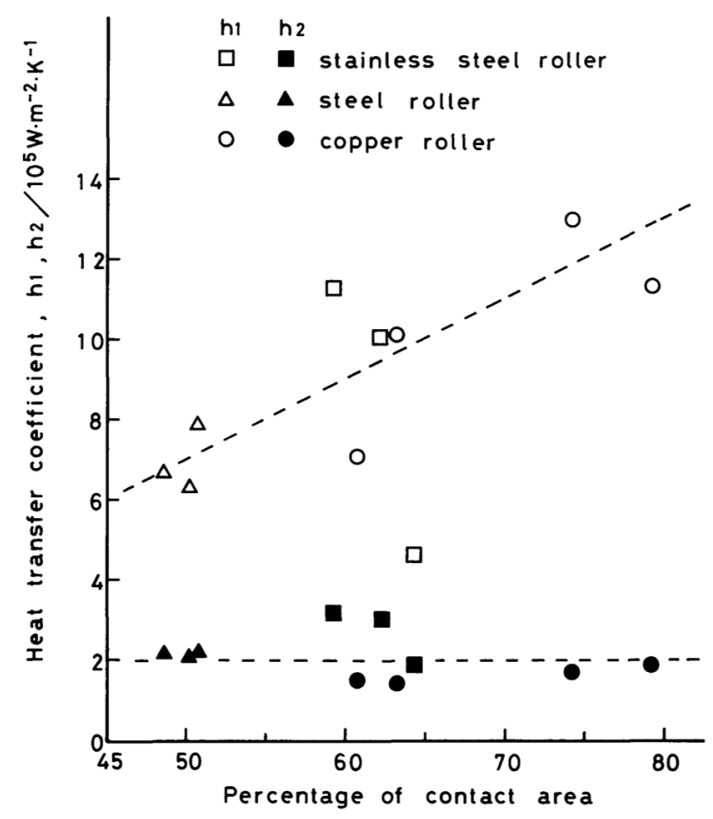

Fig. 11 Relation between the estimated values of heat transfer coefficient and contact area.

$\mathrm{W} \cdot \mathrm{m}^{-2} \cdot \mathrm{K}^{-1}$ after Davies et $a l .{ }^{(9)}$. These $h$ values appear to be quite different from one another.

However the $h_{1}$ values estimated in the pre- sent study, varying in the range from $7 \times 10^{5}$ $\mathrm{W} \cdot \mathrm{m}^{-2} \cdot \mathrm{K}^{-1}$ to $1.3 \times 10^{6} \mathrm{~W} \cdot \mathrm{m}^{-2} \cdot \mathrm{K}^{-1}$, are close to the $h$ values reported by Huang and Fiedler, and Katgerman and Brink, while the estimated $h_{2}$ value of $2 \times 10^{5} \mathrm{~W} \cdot \mathrm{m}^{-2} \cdot \mathrm{K}^{-1}$ is close to the $h$ value reported by Davies et al. That is, the variety in $h$ values reported up to the present is attributed to the characteristic of $h$ value change during the cooling by the single roller chill block casting.

Huang and Fiedler ${ }^{(7)}$, and Katgerman and Brink $^{(8)}$ have estimated the $h$ values by best fitting of the calculated results to the experimental relation between ribbon thickness and casting time, or between ribbon thickness and roller surface velocity. It is within the puddle region that they have made an analysis on the ribbon formation process in order to estimate the $h$ values. Therefore, the $h$ values estimated by them correspond to the $h_{1}$ values estimated in the present study. Rather large and widely varying $h$ values reported by Huang and Fiedler may be due to their treatment that the viscous layer, developing within the puddle region, only contributes to the resultant ribbon thickness and the contribution from the momentum boundary layer, in other words, non-viscid layer, is neglected ${ }^{(3)-(5)(8)}$.

Davies et al. ${ }^{(9)}$ have estimated the $h$ values by using an extrapolated experimental relation between dendrite arm spacing and cooling rate and by assuming Newtonian cooling condition. The dendrite arm spacing measurements have been made in the middle or near the top surface regions of a ribbon cast. Therefore, the $h$ values estimated by Davies et al. corresponds to the $h_{2}$ values estimated in the present study. Rather small $h$ values reported by Davies et al. may be due to the extrapolation of dendrite arm spacing versus cooling rate relation to higher cooling rate, for such extrapolation has been recently pointed out to be questionable ${ }^{(10)}$.

\section{Conclusion}

The effects of three different roller materials and roller surface velocity on the cooling of a $\mathrm{Ni}-19$ at $\% \mathrm{P}$ alloy during the single roller chill block casting were investigated by measuring the temperatures of solidifying ribbons with an 
infrared thermometer.

The values of heat transfer coefficient at the metal-roller interface were estimated by best fitting of the calculated results, obtained from the heat transfer analysis, to the experimentally measured temperatures of a solidifying ribbon.

The conclusion obtained from the present work is as follows:

(1) The value of heat transfer coefficient is not constant throughout the casting, but varies from the relatively large $h_{1}$ value to the small $h_{2}$ value.

(2) Though such process variables as roller material and roller surface velocity affect the contact condition between ribbon and roller surface, the estimated $h_{1}$ values increase in the range from $7 \times 10^{5} \mathrm{~W} \cdot \mathrm{m}^{-2} \cdot \mathrm{K}^{-1}$ to $1.3 \times 10^{6}$ $\mathrm{W} \cdot \mathrm{m}^{-2} \cdot \mathrm{K}^{-1}$ with the increase only in contact area from $50 \%$ to $80 \%$. The estimated $h_{2}$ value is, however, almost constant, being $2 \times 10^{5}$ $\mathrm{W} \cdot \mathrm{m}^{-2} \cdot \mathrm{K}^{-1}$ independent of contact area.

\section{Acknowledgement}

This work was supported in part by the
Grant in Aid for Scientific Research through the Ministry of Education, Science and Culture, Japan.

\section{REFERENCES}

(1) S. Kavesh: Metallic Glasses, ASM, (1978), p. 36.

(2) H. Hillmann and H. R. Hilzinger: Rapidly Quenched Metals III, The Metals Society, (1978), p. 22.

(3) L. Katgerman: Scripta Met., 14 (1980), 861.

(4) P. H. Shingu, K. Kobayashi, R. Suzuki and K. Takeshita: Rapidly Quenched Metals IV, The Japan Inst. Metals, (1982), p. 57.

(5) K. Takeshita and P. H. Shingu: Trans. JIM, 24 (1983), 529.

(6) R. C. Ruhl: Mater. Sci. Eng., 1 (1967), 313.

(7) S. C. Huang and H. C. Fiedler: Mater. Sci. Eng., 51 (1981), 39.

(8) L. Katgerman and P. J. Van den Brink: Rapidly Quenched Metals IV, The Japan Inst. Metals, (1982), p. 61 .

(9) H. A. Davies, N. Shohoji and D. H. Warrington: Rapid Solidification Processing II, claitor's pub. div., (1980), p. 153.

(10) R. Sellger and W. Löser: Cryst. Res. Tech., 16 (1981), 1181 . 